\title{
The Effect of Polymer Immobilization on Magnetic Properties of Magnetosomes
}

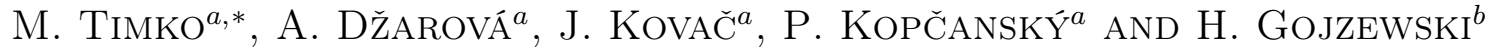 \\ ${ }^{a}$ Institute of Experimental Physics, Slovak Academy of Sciences, Watsonova 47, 04001 Košice, Slovakia \\ ${ }^{b}$ Institute of Physics, Poznań University of Technology, Nieszawska 13A, 60-965 Poznań, Poland \\ The anisotropy of several PVA/magnetosomes magnetically textured films has been investigated from the \\ point of view of magnetic properties. The thin film synthesized under the influence of a magnetic field (99 mT) \\ shows the typical behavior of an anisotropic material. On sample textured in magnetic field the magnetisation \\ loop measurements were performed in dependence on the direction of the external magnetic field with respect \\ to the texture axis. The results of hysteresis measurements show that the magnetic field applied during the \\ synthesis promotes an orientation of the chains of magnetosomes and permanently textured film is obtained \\ after solidification. The obtained values for magnetic remanence and coercivity were dependent on the external \\ magnetic field orientation and the alignment effect of particle moments may be clearly seen which is in agreement \\ with the theoretical model of magnetic particle chains.
}

PACS numbers: 75.30.Cr, 75.30.Gw, 75.50.Mm, 75.50.Tt, 75.60.Ej

\section{Introduction}

The magnetotactic bacteria are well known for their magnetotaxes orienting the cells along the geomagnetic field [1]. The tactic behavior of these bacteria is caused by inner magnetosome chains consisting of single domain magnetite nanoparticles. Magnetosomes, which can be isolated from microbial cells in larger quantities, consist of a magnetic mineral crystal, magnetite or greigite, enveloped by a biological membrane that contains phospholipids and specific proteins [2]. The magnetosome membrane is not only critical for the control of crystal size and morphology, but also prevents the aggregation of extracted magnetosomes and thus stabilizes magnetosome suspensions. Although variations exist between species almost all magnetosomes fall in the narrow size range of $35-120 \mathrm{~nm}$ when measured along their longest axes [3]. This size range is significant as places these particles within the stable magnetic single domain particles. The magnetic particles in this size range are uniformly magnetized and their remanent magnetic moment is maximally equal to the saturation magnetic moment. Magnetic nanoparticles smaller than $30 \mathrm{~nm}$ show the superparamagnetic behavior. These particles are uniformly magnetized, too, but their moment is not constant in direction because of thermal induced reversals which produce the time averaged moment of zero. Therefore, the magnetosomes have optimum size for maximum moment per particle [4]. During the past decades progress has been made in investigation of magnetosome biomineralization process [5] but the understanding of the mechanism of magnetosome formation and specific steps in-

\footnotetext{
* corresponding author; e-mail: timko@saske.sk
}

volved in the biomineralization process are still under dispute. The unique structure and magnetic properties of magnetosome chains represent the high potential in various biomedical and technological applications. They can, for instance, be inserted in a silica matrix to form a composite magneto-optical material which can be easily used in integrated optical devices [6]. One of the potential application areas of magnetosomes is magnetic particle hyperthermia and magnetic drug targeting [7]. The purpose of this work is to study the magnetic properties of magnetically textured polymer films doped by magnetosomes.

\section{Experimental}

Bacterial magnetosomes investigated in this contribution were obtained by mineralization process of a Gram-negative $\alpha$-proteobacterium Magnetospirillum strain AMB-1. This bacteria is known as a more oxygen-tolerant bacteria. The detailed description of the cultivation process of magnetotactic bacteria and the isolation of magnetosomes from bacteria is given in our previous contribution [8]. For the isolation of the magnetosome particles, we have used the modified method described by Grünberg et al. [9]. For the isolation of magnetosomes approximately $10 \mathrm{~g}$ (wet weight) cells of Magnetotacticum Magnetospirillum suspended in $100 \mathrm{ml}$ of 20 mM HEPES-4 mM EDTA, pH 7.4, was split up (disrupted) by sonification. The unbroken cells and the cell debris were removed from the sample by centrifugation $(10 \mathrm{~min}, 3036 \mathrm{rpm})$. The cell extract was placed onto a permanent magnet (NdFeB-magnets, approximately $1 \mathrm{~h}$ ). The black magnetosomes sedimented at the bottom of the tube and the residual contaminating cellular material was decanted. To eliminate the electrostat- 
ically bound contamination, the magnetic particles were rinsed first with $50 \mathrm{ml}$ of $10 \mathrm{mM}$ HEPES-200 $\mathrm{mM} \mathrm{NaCl}$, $\mathrm{pH}$ 7.4, and subsequently with $100 \mathrm{ml}$ of $10 \mathrm{mM}$ HEPES, $\mathrm{pH}$ 7.4. After removal of the cell extract from the magnets, the magnetic particles were flushed with $10 \mathrm{mM}$ HEPES buffer. The magnetosome suspension (black sediment) was centrifugated (18000 rpm, $30 \mathrm{~min}$ at $\left.4^{\circ} \mathrm{C}\right)$. After centrifugation the cell extract was placed on the magnet for $30 \mathrm{~min}$. The last procedure was repeated ten times to obtain well purified magnetosomes.

Magnetosome films are bacterial magnetic particles embedded in polyvinyl acetate (PVA) polymer matrix. Process of the preparation of thin films was based on blending of some mg magnetosomes with $1.5 \mathrm{mg}$ of PVA $0.5 \%$. The composite PVA/magnetosomes suspension was coated as films on the Petri bowls. The gelation of the films was obtained by drying in air. These films were prepared under the influence of a magnetic field or without any field. We have used homogeneous field intensity of $99 \mathrm{mT}$.

The morphological properties and the size of magnetosomes were estimated by transmission measurements using FEI Tecnai F20 S-TWIN (Philips Corp.) transmission electron microscope (TEM) working at the accelerating voltage of $200 \mathrm{kV}$. The magnetization properties of the prepared magnetosome films in perpendicular and parallel configuration relative to applied magnetic field during solidification were measured by SQUID magnetometer of Quantum Design in a magnetic field (up to $2 \mathrm{~T}$ ) and in a temperature range $2-300 \mathrm{~K}$.

\section{Results and discussion}

The typical result from TEM is presented in Fig. 1. As it was observed previously in our work [8] here also the micrographs obtained from TEM by replication tech-

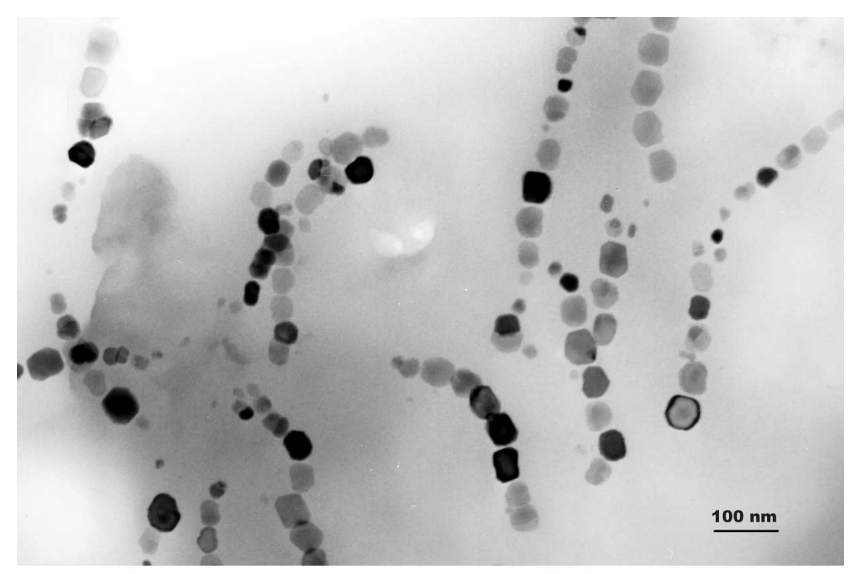

Fig. 1. Transmission electron micrograph of magnetosomes.

nique, showed that in our sample the magnetosomes after isolation are arranged in bent chains to minimize they stray field energy. The mean size and standard devia-

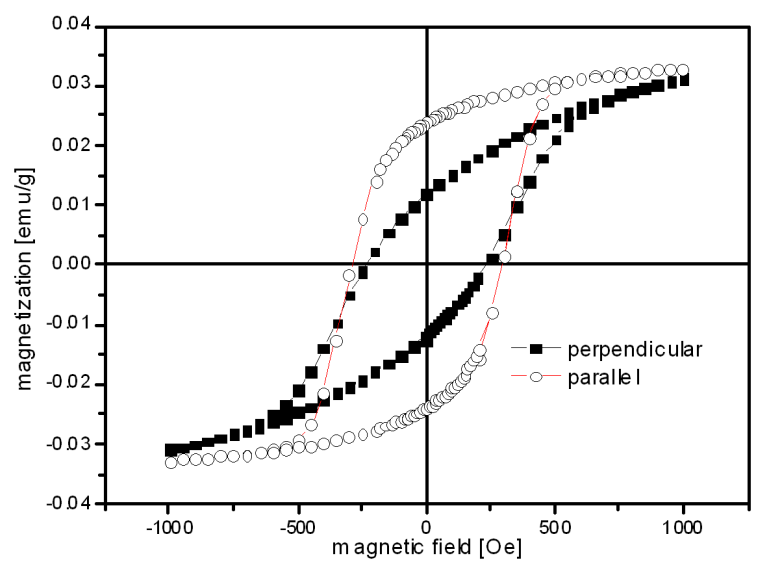

Fig. 2. Magnetic hysteresis loops of textured samples of magnetosomes in dependence on the direction of the external magnetic field with respect to texture axis.

tion estimated from TEM was $34 \mathrm{~nm}$ and $6 \mathrm{~nm}$, respectively. For aqueous suspension of investigated magnetosomes the coercivity at room temperature was $18.5 \mathrm{Oe}$ and remanent magnetization $21 \mathrm{emu} / \mathrm{g}$. As it can be seen from Fig. 2 after solidification magnetosomes in PVA with application of external magnetic field (99 $\mathrm{mT})$ the coercivity increased to 310 Oe for parallel orientation and 251 Oe for perpendicular orientation of external magnetic field to the texture axis using a constant magnetic field of $99 \mathrm{mT}$. The anisotropy of coercivity matches well the theoretical model of magnetic chains [10]. From measurements of aqueous suspension the ratio of remanent and saturation magnetization $\left(M_{\mathrm{r}} / M_{\mathrm{s}}\right)$ was estimated to be 0.47 , which is in excellent agreement with the theoretical value of 0.5 for a random dispersion of single-magnetic-domain particles. After solidification process the values of $\left(M_{\mathrm{r}} / M_{\mathrm{s}}\right)$ were 0.84 for parallel and 0.32 for perpendicular orientation which clearly showed to the alignment effect of magnetosome chains. In our previous work [11] the alignment effect was studied by the magneto-optical measurement. In this experiment the modification of the state of polarization was measured using an ellipsometer and the ellipsometric angles and then the anisotropy were determined. The magnetic field applied during the gelation of the polymer matrix has provided an orientation of the optical axis of the magnetosomes chains. This anisotropy was linked to the body of the particles, which is blocked after the gelation, the anisotropy remains even if the field is switch off. Thus, the polymer films behave as a permanent anisotropic material suitable for the realization of self-polarized magneto-optic devices.

\section{Conclusion}

The results from magnetic measurements showed that the magnetic field applied during the synthesis promotes an orientation of the chains of magnetosomes. Thus, after solidification, a permanent textured film was obtained. 


\section{Acknowledgments}

This work was supported by Slovak Academy of Sciences, in the framework of CEXNANOFLUID, and Ministry of Education Agency for structural funds of EU in frame of projects Development of technology of magnetic fluids for biomedical applications, project No. 26220220005 .

\section{References}

[1] R. Blakemore, Science 190, 377 (1975).

[2] D.L. Balkwill, D. Maratea, R.P. Blakemore, J. Bacteriol. 141, 1399 (1980).

[3] D.A. Bazylinski, A.J. Garratt-Reed, R.B. Frankel, Microsc. Res. Technol. 27, 389 (1994).

[4] R.B. Frankel, D.A. Bazylinski, D. Schuler, Supramol. Sci. 5, 398 (1998).
[5] R.B. Frankel, D.A. Bazylinski, Nat. Rev. Microbiol. 2, 217 (2004).

[6] F. Choueikani, F. Royer, D. Jamon, A. Siblini, J.J. Rousseau, S. Neveu, J. Charara, Appl. Phys. Lett. 94, 051113 (2009).

[7] P. Moroz, S.K. Jones, B.N. Gray, Int. J. Hyperthermia 18, 267 (2002).

[8] M. Timko, A. Džarová, V. Závišová, M. Koneracká, A. Sprincová, P. Kopčanský, J. Kováč, I. Vávra, A. Szlaferek, Magnetohydrodynamics 44, 113 (2008).

[9] K. Grünberg, C. Wawer, B.M. Tebo, D. Schüler, Appl. Environ. Microbiol. 67, 4573 (2001).

[10] E. Blums, A. Cebers, M. Maiorov, Magnetic Fluids, de Gruyter, Berlin 1997, p. 18.

[11] A. Dzarova, F. Royer, D. Jamon, M. Timko, P. Kopcansky, H. Gojzewski, F. Choueikani, J.J. Rousseau, J. Magn. Magn. Mater., accepted for publication. 